Article

\title{
Nonlinear Viscoelastic-Plastic Creep Model Based on Coal Multistage Creep Tests and Experimental Validation
}

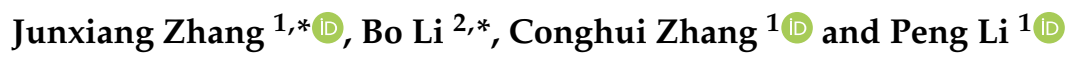 \\ 1 School of Energy \& Environment, Zhongyuan University of Technology, Zhengzhou 450000, China \\ 2 College of Safety Science and Engineering, Henan Polytechnic University, Jiaozuo 454000, China \\ * Correspondence: 6782@zut.edu.cn (J.Z.); anquanlibo@163.com (B.L.); \\ Tel.: +86-1510-371-3773 (J.Z.); +86-1383-911-7169 (B.L.)
}

Received: 22 August 2019; Accepted: 3 September 2019; Published: 9 September 2019

\begin{abstract}
The development of fractures, which determine the complexity of coal creep characteristics, is the main physical property of coal relative to other rocks. This study conducted a series of multistage creep tests to investigate the creep behavior of coal under different stress levels. A negative elastic modulus and a non-Newtonian component were introduced into the classical Nishihara model based on the theoretical analysis of the experimental results to propose a nonlinear viscoelastic-plastic creep model for describing the non-decay creep behavior of coal. The validity of the model was verified by experimental data. The results show that this improved model can preferably exhibit decelerating, steady state, and accelerating creep behavior during the non-decay creep process. The fitting accuracy of the improved model was significantly higher than that of the classical Nishihara model. Given that acceleration creep is a critical stage in predicting the instability and failure of coal, its successful description using this improved model is crucial for the prevention and control of coal dynamic disasters.
\end{abstract}

Keywords: multistage creep test; negative elastic modulus; non-Newtonian component; non-decay creep; accelerating creep

\section{Introduction}

With the gradual depletion of mineral resources in the shallow, most mines begin the trend of deep mining [1,2]. The rheological characteristics of coal become more significant with the increase in strata stress, thereby decreasing its mechanical strength under long-term static load, which causes deep mining to face many time-related problems [3,4], such as aggravated roadway deformation, collapse of gas extraction boreholes, and gas and coal outburst delay [5-7]. All of these problems pose serious threats to underground safety. Therefore, exploring the creep characteristics of coal and presenting an applicable creep model are crucial for the underground engineering design, mining construction, and safe production of coal mines.

Research methods for the rheological model are mainly divided into empirical equation and component combination [8,9]. Logarithmic, exponential, and power functions are adopted in the empirical equation to describe the strain-time relation with few fitting parameters and strong pertinence based on the creep test results. However, this method has some defects, such as long experiment time, unclear physical meaning, and difficult extrapolation of research results $[10,11]$. The component combination method uses Hooker $(\mathrm{H})$, Newton $(\mathrm{N})$, and plastic $(\mathrm{Y})$ units to establish rheological constitutive models, such as Burgers and Nishihara models, to reflect the rheological behavior of rocks with clear physical significance. However, these models cannot reproduce the accelerating creep stage 
because of the use of linear components in the models [12]. Shao et al. [13,14] proposed constitutive models that could represent the induced plastic deformation, damage, and creep characteristics of brittle rocks to describe nonlinear creep characteristics. Ping [15] developed a new nonlinear-damage creep constitutive model that combined the Burgers, Hooke, and St. Venant models, and the applicability of this model was verified by a series of uniaxial compression creep tests. Kang [16] established a fractional nonlinear creep model that considered the viscoelastic-plastic characteristic and damage effects of coal; moreover, he also conducted several uniaxial creep tests under different axial stress conditions to verify the model. Cai $[17,18]$ presented an improved hardening damage creep model by introducing hardening and damage functions based on the uniaxial creep test of lean coal. Furthermore, Wang [19] deduced a nonlinear creep constitutive model of coal under three-dimensional (3D) stress condition and achieved satisfactory fitting results with the experimental data of coal unloading confining pressure creep.

Coal, a natural organic mineral, has undergone a long geological period of evolution and is affected by mining stress, which results in the generation of abundant fractures with poor homogeneity inside the coal, and determines the complexity of its creep behavior under different loads. However, only limited research has focused on the creep characteristics of coal. Thus, a suitable creep model must be proposed. Motivated by the aforementioned facts, we conducted a series of multistage creep tests on coal specimens and analyzed coal creep behaviors under different loading conditions to develop a new nonlinear viscoelastic-plastic creep model using empirical equations and component combinations. The accuracy and applicability of this model were verified by model identification, and the effects of model parameters on creep behavior were also discussed in detail. The results obtained in this work are expected to provide some scientific basis for the study of coal instability failure and dynamic disasters.

\section{Multistage Creep Tests}

High development degree of microfissures (with the aperture less than $0.1 \mathrm{~mm}$ ), which is the principal factor that affects coal creep behavior, is the main physical characteristic of coal. Figure 1 shows the micromorphology of anthracite coal samples collected from Jiulishan Coal mine using a JSM-6390/LV scanning electron microscope (SEM), where several fractures can be clearly observed. The data of the industrial analysis and basic parameters of coal sample are also listed in Table 1. These fractures are affected by the influence of coal-forming and geological processes, and a large number of intricate fissure networks were generated. Stress concentration will be firstly formed around fractures during the loading process, thereby causing local damage and inhomogeneous deformation inside the coal, and further inducing a weakening effect on the mechanical strength. When this weakening effect accumulates to a certain extent, the fractures will expand rapidly, thereby accelerating the instability and failure of the coal. Especially for newly exposed coals affected by pressure relief, massive secondary fractures were generated and resulted in significant creep characteristics. To determine the special physical properties of coal, this study performed a series of triaxial compression creep tests with stepwise loading on coal specimens and analyzed the creep laws under different stress levels to introduce a new model suitable for describing coal creep behavior.

Table 1. Industrial analysis and basic parameters of coal sample.

\begin{tabular}{cccc}
\hline Parameter & Value & Parameter & Value \\
\hline Adsorption constant a & $29.412 \mathrm{~m}^{3} / \mathrm{t}$ & $M_{\mathrm{ad}}$ & $0.44 \%$ \\
Adsorption constant $\mathrm{b}$ & $2.252 \mathrm{MPa}^{-1}$ & $A_{\mathrm{d}}$ & $8.12 \%$ \\
True density & $1.56 \mathrm{~g} \cdot \mathrm{cm}^{-3}$ & $V_{\mathrm{daf}}$ & $10.58 \%$ \\
Apparent density & $1.49 \mathrm{~g} \cdot \mathrm{cm}^{-3}$ & $F C_{\mathrm{ad}}$ & $80.86 \%$ \\
\hline
\end{tabular}




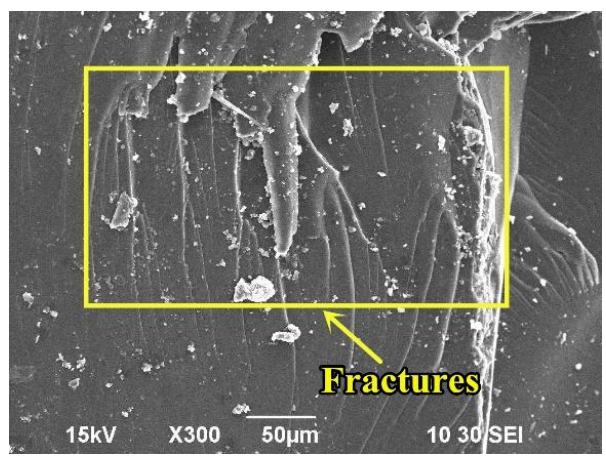

Figure 1. Micromorphology of coal sample.

\subsection{Specimen Preparation and Experimental Set-Up}

The coal samples used in the multistage creep tests were selected from an underground mining face in Jiulishan Coal mine (Figure 2a,b). In accordance with the experimental specification recommended by the International Society for Rock Mechanics (ISRM) [20], the specimens were cored from intact coal blocks and prepared to the standard cylindrical dimensions with a diameter and height of $50 \mathrm{~mm}$ and $100 \mathrm{~mm}$, respectively (Figure 2c). The triaxial creep tests were performed on the RLW-500G coal-rock triaxial creep experimental system (Figure 2d), which is equipped with features, such as axial loading, confining pressure loading, pore pressure loading, servo system, control system, data acquisition, and automatic drawing system. The digital servo controller is used in the control part of the axial loading and confining pressure loading systems, which can automatically complete the triaxial creep tests.
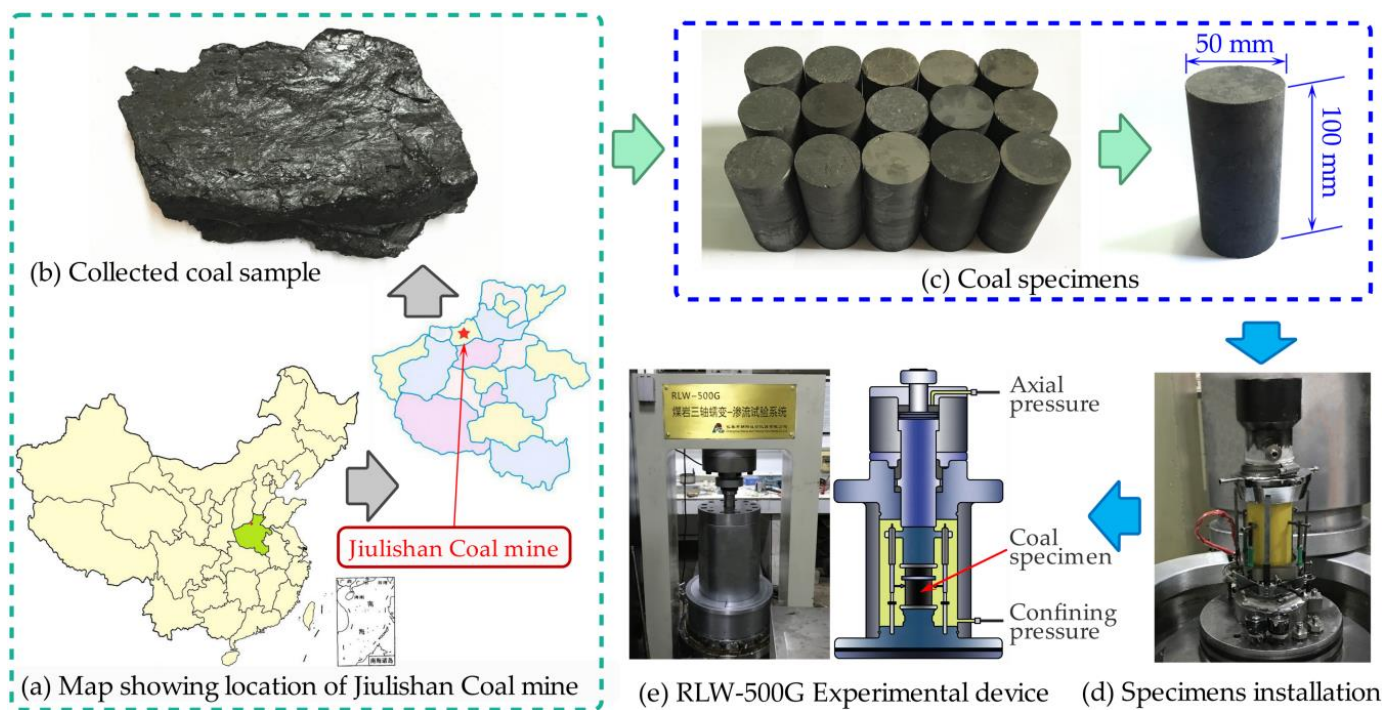

Figure 2. Specimen preparation and experimental device.

The main technical parameters of the RLW-500G experimental device are presented as follows:

(a) The main engine adopts a framework structure with stiffness of more than $5000 \mathrm{kN} / \mathrm{mm}$.

(b) The maximum axial capacity is $500 \mathrm{kN}$, with measure resolution and precision less than $2.5 \mathrm{~N}$ and $1 \%$, respectively. The maximum confining pressure is $50 \mathrm{MPa}$, with a measure resolution and precision of less than $0.001 \mathrm{MPa}$ and $\pm 1 \%$, respectively.

(c) Axial and radial extensometer deformation measuring ranges are 0-15 $\mathrm{mm}$ and $0-7 \mathrm{~mm}$, respectively; and the measuring accuracy is $\pm 0.5 \%$.

(d) Limit control system: When the parameters of axial deformation, radial deformation, or test time reach a preset value, or the specimen split, oil pipe blockage, and oil temperature too high, the device can be automatically stopped for self-protection. 


\subsection{Experimental Scheme}

The loading methods used in the creep test include single-stage and multistage loading. Multistage loading, which has the advantages of convenient operation and a high adoption rate, was applied in this work for the triaxial compressive creep tests of coal specimens.

Because the low gas pressure and small confining pressure of collected coal sample taken from the mining face in the Jiulishan mine are low, the experimental conditions were set during the experimental process to simulate the real underground field conditions: $1 \mathrm{MPa}$ confining pressure, $0.2 \mathrm{MPa}$ gas pressure, and $25^{\circ} \mathrm{C}$ ambient temperature. The specific experimental procedures are presented as follows:

First, initial axial and confining pressures were applied to $1 \mathrm{MPa}$ at a rate of $200 \mathrm{~N} / \mathrm{s}$. Second, the cylinder intake valve was opened to introduce methane gas into the triaxial-gripper of the specimen until the inlet pressure reached the preset experimental value. Third, the adsorption state was maintained for $24 \mathrm{~h}$ to simulate the original adsorption of gas-filled coal. Subsequently, axial stress was loaded at a rate of $100 \mathrm{~N} / \mathrm{s}$ to the preset value of $5 \mathrm{MPa}$. Each axial stress stage was maintained for a sufficient time until axial strain trended to be stable. Afterwards, the next stage of axial stress was loaded again, in which the gradient of axial compression is $10 \mathrm{MPa}$, until the specimen failed. Finally, the next coal specimen was replaced for testing. The axial strain data were recorded during the experiment.

\subsection{Experimental Results}

The creep curves of increased deviatoric stress gradings on coal specimens can be obtained from multistage loading creep tests. However, the experimental results present differences owing to the complexity of fractures in different coal specimens. Therefore, JC-1 and JC-2, which are the most representative coal specimens, are analyzed in Figure 3.
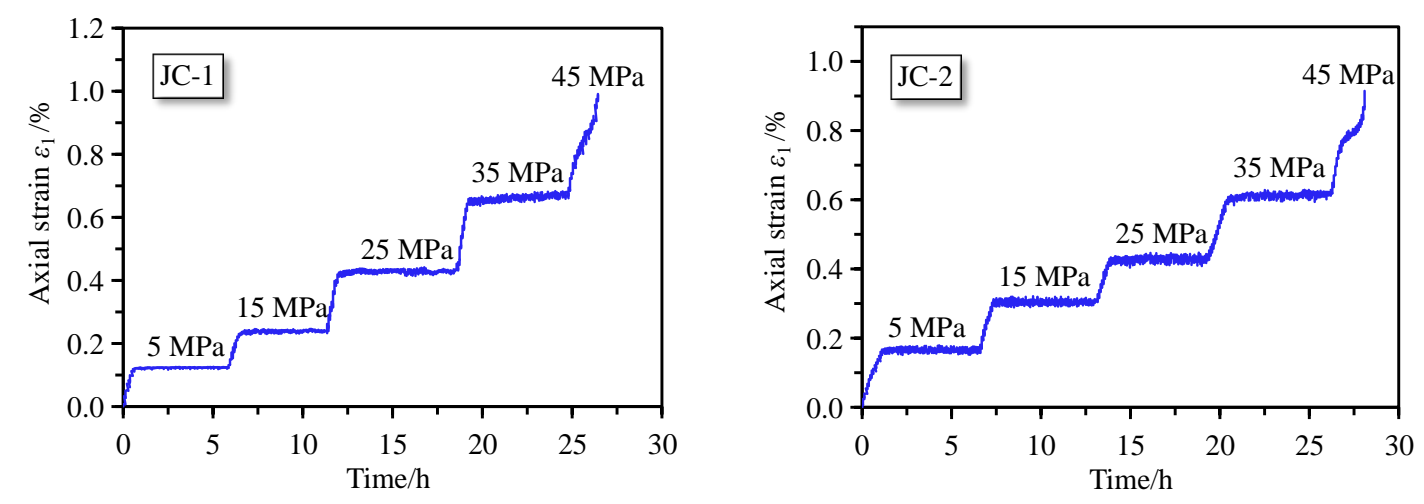

Figure 3. Creep testing curves of different coal specimens.

To further investigate the creep behavior of coal specimens under different stress levels, the whole creep curves were transformed into multiple curves with various creep loads according to the data processing method for multistage loading creep test proposed on the basis of comprehensive superposition principle [21]. The results are shown in Figure 4.

Figure 4 shows that the creep characteristics of coal specimens greatly varied under different stress levels. Axial strain continuously increased with stress during the graded loading process. The test results show that when stress was less than $35 \mathrm{MPa}$, the creep rate of specimens JC- 1 and JC-2 (i.e., both underwent decelerating and steady state creeps) decreased with time. Finally, coal strain tended to a certain value. When the load reached $45 \mathrm{MPa}$, the creep rate decreased first and then increased with time. For example, specimen JC-1 was developed directly from decelerating creep to accelerating creep stage at $1.0 \mathrm{~h}$. Specimen JC-2 also experienced a short period of steady state creep and swiftly developed into accelerating creep. During the accelerating creep, the coal specimens easily 
exhibited inconsistent deformations owing to the sharp increase in axial strain, thereby aggravating the formation and convergence of fractures inside the coal, leading to the complete failure of the whole specimen. The failure characteristics of the coal specimens in Figure 5 show that the failure of coal specimens was mainly caused by a dominant crack that split the whole specimen into several thin sheets. Therefore, the monitoring of coal creep characteristics in the process of underground mining is of great engineering significance.
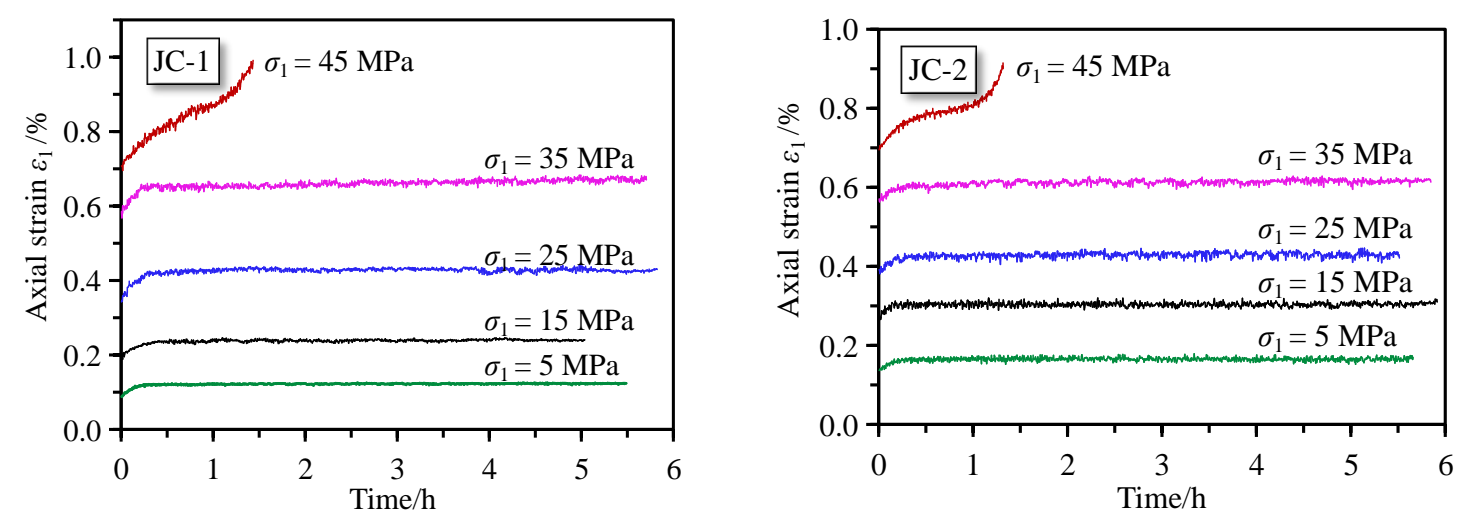

Figure 4. Creep testing curves under different stress levels.
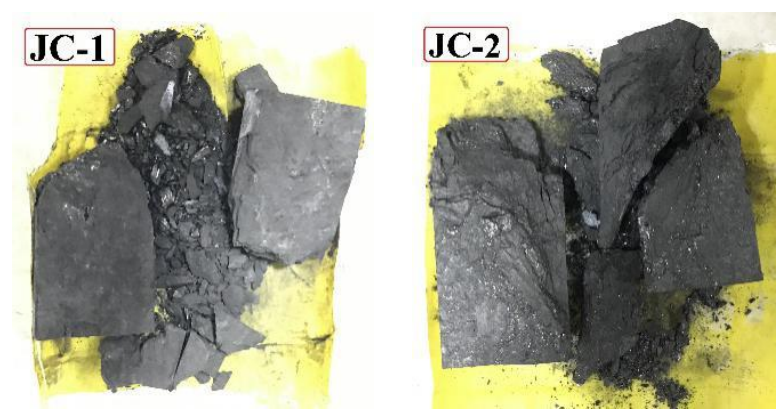

Figure 5. Failure characteristics of coal specimens

The multistage creep test results show that the coal creep characteristics under different stress levels are significant. According to the stress on coal specimen, the creep types can be divided into decay and non-decay creeps (Figure 6).

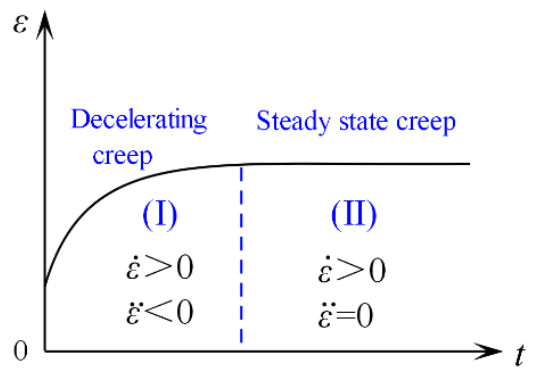

(a) Decay creep

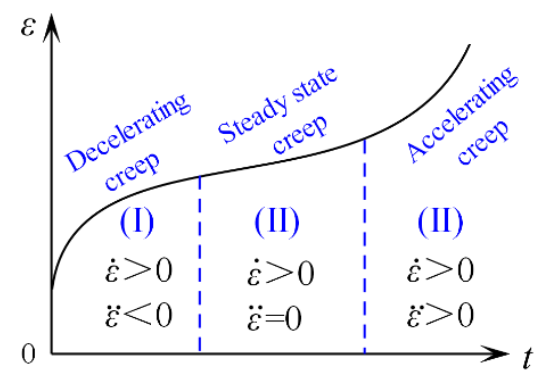

(b) Non-decay creep

Figure 6. Creep characteristic of coal specimens.

If the stress level is less than the yield stress of the coal, then coal presents decay creep, which is only characterized by decelerating (I) and steady state creep (II). However, when the stress level is larger than the yield stress, the coal exhibits non-decay creep performance, which includes decelerating (I), steady state (II), and accelerating (III) creep stages. 
(1) In decelerating creep, creep rate decreases with the increase in axial strain, that is, creep rate $\dot{\varepsilon}>0$ and creep acceleration $\ddot{\varepsilon}<0$. The creep curve in this stage presents a convex shape.

(2) In steady state creep, the coal strain tends to be constant along with time, that is, creep rate $\dot{\varepsilon}>0$ and creep acceleration $\ddot{\varepsilon}=0$, with a linear change tendency of creep curve.

(3) In accelerating creep, when the coal strain goes beyond the yield point, the axial strain and creep rate increase rapidly, that is, creep rate $\dot{\varepsilon}>0$ and creep acceleration $\ddot{\varepsilon}>0$, and the creep curve is presented as a concave shape.

The aforementioned analysis shows that the convex-concave features of a coal creep curve will be changed only when non-decay creep occurs. Therefore, the coal creep types can be recognized by the convex-concave variation. We assume that the coal strain $\varepsilon(t)$ is a function of time. Thus, if the inflection point of the creep curve does not appear, then only decelerating and steady state creep occur, and the curve is convex. The coal will only enter the accelerating creep stage when the inflection point appears. At this time, the concave-convex property of the creep curve also changes. Therefore, the existence of an inflection point in the creep curve is a necessary and sufficient condition to judge the occurrence of accelerating creep of coal. If the occurrence time of the inflection point can be accurately predicted, then some effective protective measures can be considered in time to control the potential safety hazards of coal instability and failure. For this reason, finding the creep inflection point by establishing a creep mathematical model that can describe the nonlinear change characteristic of coal strain with time is important.

\section{Analysis of Classical Nishihara Model}

As for the study in the creep models of coal and rock, the classical Nishihara model (Figure 7), which consists of a generalized Kelvin and ideal viscoplastic body, is characterized by a simple model structure and can fully reflect the elastic-viscoelastic-viscoplastic characteristics of rock. Therefore, the model has been widely used in rock rheology.

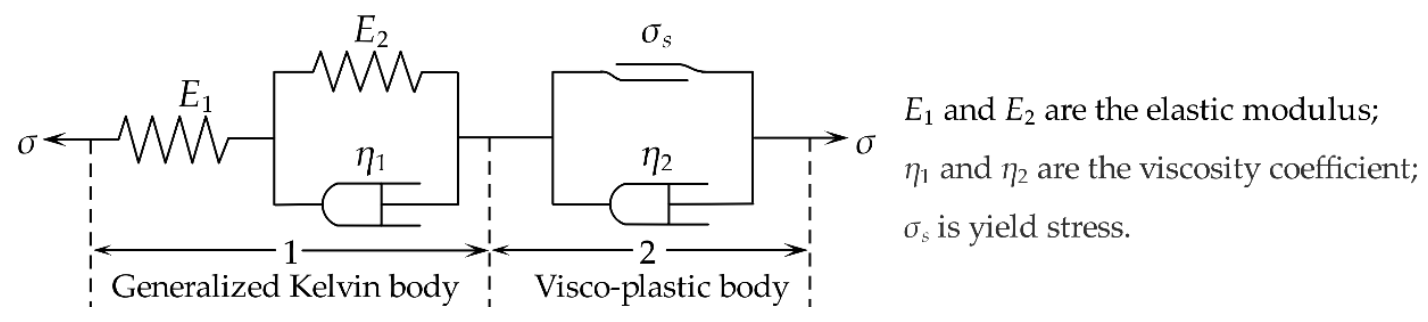

Figure 7. Classical Nishihara model.

The Nishihara model is only suitable for describing the decelerating and steady state creep characteristics of soft rock, because all the components used in this model are linear [22,23]. We assume that the volume change of coal is elastic, and coal deformation is caused by deviatoric stress. Thus, if deviatoric stress is less than yield stress, that is, $\left(\sigma_{1}-\sigma_{3}\right)<S_{h}$, then the creep equation of the classical Nishihara model under equal confining pressure conditions is given by the following [24]:

$$
\varepsilon=\frac{\left(\sigma_{1}-\sigma_{3}\right)}{3 G_{1}}+\frac{\left(\sigma_{1}-\sigma_{3}\right)}{3 G_{2}}\left[1-\exp \left(-\frac{G_{2}}{\eta_{1}} t\right)\right]+\frac{\left(\sigma_{1}-\sigma_{3}\right)-S_{h}}{3 \eta_{2}} t, \quad\left(\sigma_{1}-\sigma_{3}\right)<S_{h},
$$

where $\varepsilon$ is the strain of the model; $\sigma_{1}$ and $\sigma_{3}$ are the first and third principal stresses, respectively; $G_{1}$ and $G_{2}$ are the shear modulus of generalized Kelvin and ideal viscoplastic body, respectively; $\eta_{1}$ and $\eta_{2}$ are the viscosity coefficients; $S_{h}$ is the deviatoric yield stress of the model; and $t$ is the creep time.

In accordance with Equation (1), the analytical software MATLAB was used to fit the creep test data of coal specimens with decay creep characteristic under $35 \mathrm{MPa}$. Table 2 shows the fitting results of the model parameters, and Figure 6 depicts the comparison results between fitting curves and test data. 
Table 2. Parameter values of classical Nishihara model.

\begin{tabular}{cccccc}
\hline Specimen & $\sigma_{\mathbf{1}} / \mathbf{M P a}$ & $G_{\mathbf{1}} / \mathbf{G P a}$ & $G_{\mathbf{2}} / \mathrm{GPa}$ & $\left.\eta_{\mathbf{1}} / \mathbf{( G P a} \cdot \mathbf{h}\right)$ & $\boldsymbol{R}_{\mathbf{2}}$ \\
\hline \multirow{3}{*}{$\mathrm{JC}-1$} & 5 & 15.049 & 39.493 & 5.339 & 0.963 \\
& 15 & 24.120 & 102.695 & 18.413 & 0.969 \\
& 25 & 23.091 & 97.723 & 16.608 & 0.956 \\
& 35 & 18.025 & 286.917 & 352.267 & 0.934 \\
\hline \multirow{3}{*}{$\mathrm{JC}-2$} & 5 & 9.814 & 44.575 & 6.066 & 0.937 \\
& 15 & 17.452 & 128.684 & 9.252 & 0.925 \\
& 25 & 20.630 & 194.027 & 33.941 & 0.931 \\
& 35 & 19.599 & 317.160 & 136.092 & 0.953 \\
\hline
\end{tabular}

Table 2 shows that all coefficients of determination $R^{2}$ of the model parameters are above 0.925 , which indicates that the classical Nishihara model is well fit to the data of decay creep of coal. The data from Table 2 are substituted into Equation (1) to obtain the creep curves of coal specimens (Figure 8). The fitting curves coincide with the creep test data, indicating that the classical Nishihara model can describe the decelerating and steady state creep behavior satisfactorily. Thus, fitting decay creep characteristic using the classical Nishihara model is appropriate.
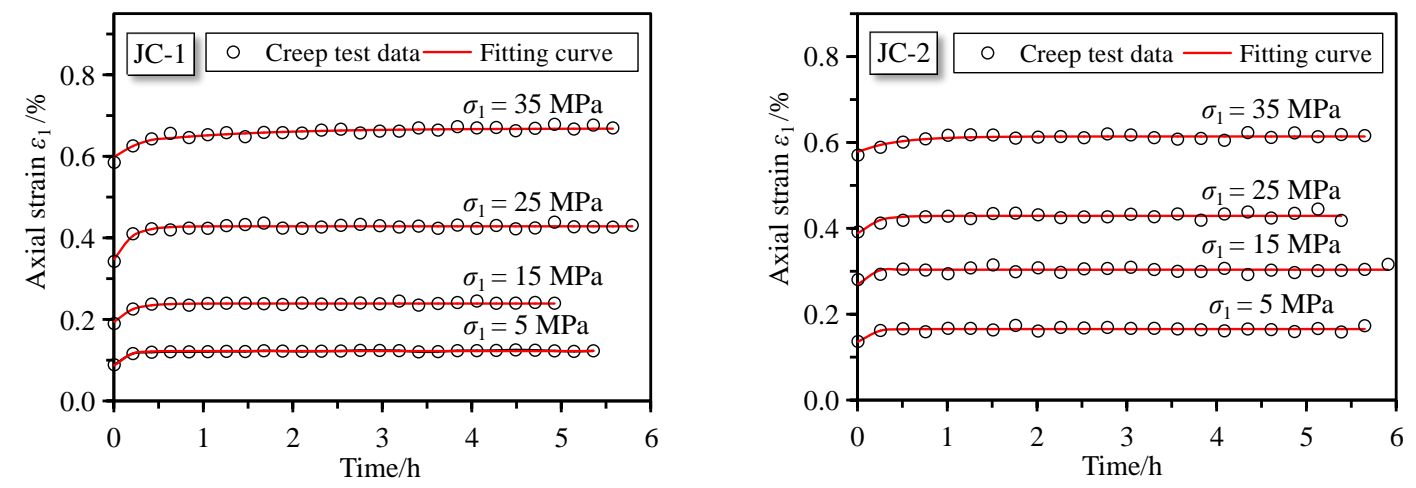

Figure 8. Comparison between fitting curves and creep test data of coal specimens.

\section{Establishment of Nonlinear Viscoelastic-Plastic Creep Model}

Considering that linear units are used in the classical Nishihara model, it cannot accurately reflect the nonlinear increase in strain with time in the accelerating creep stage. At present, the nonlinear creep model can be established using two approaches. One is the nonlinear revision of the traditional linear component, and the other is to build a nonlinear relationship between strain and time using damage mechanics or fracture mechanics theory. This study selected the first method to propose a new nonlinear viscoelastic-plastic creep model to enhance the applicability of the creep model in reflecting coal creep behavior. The concrete ideas are presented as follows:

At first, the accelerating creep curve was analyzed by empirical formula, and a non-Newtonian component with exponential variation law was presented. The non-Newtonian component was connected with elastic and plastic components in parallel by stress triggering to compose a nonlinear viscoelastic-plastic body to reflect the creep characteristic of coal in the accelerating creep stage. Subsequently, it was replaced with the ideal viscoplastic body in the classical Nishihara model to establish the nonlinear creep model.

\subsection{Model Development}

\subsubsection{Improvement of Non-Newtonian Component}

Remarkable nonlinear change tendencies that existed between coal strain and creep time were detected through the creep tests. Therefore, a non-Newtonian component can be used to characterize 
the direct proportional relation between stress and strain acceleration. Here, we used the exponential function to fit the creep test data of the accelerating creep stage, and the function equation is described as follows:

$$
\varepsilon=\varepsilon_{0}+A \exp (n t),
$$

where $\varepsilon_{0}$ is the initial strain of the accelerating stage; $A$ is the fitting parameter; and $n$ is the acceleration index, $n>0$.

The comparison results between the test data and fitting curves of accelerated creep stage of coal specimens shown in Figure 9 can be obtained on the basis of Equation (2), and the parameter fitting results are listed in Table 3.
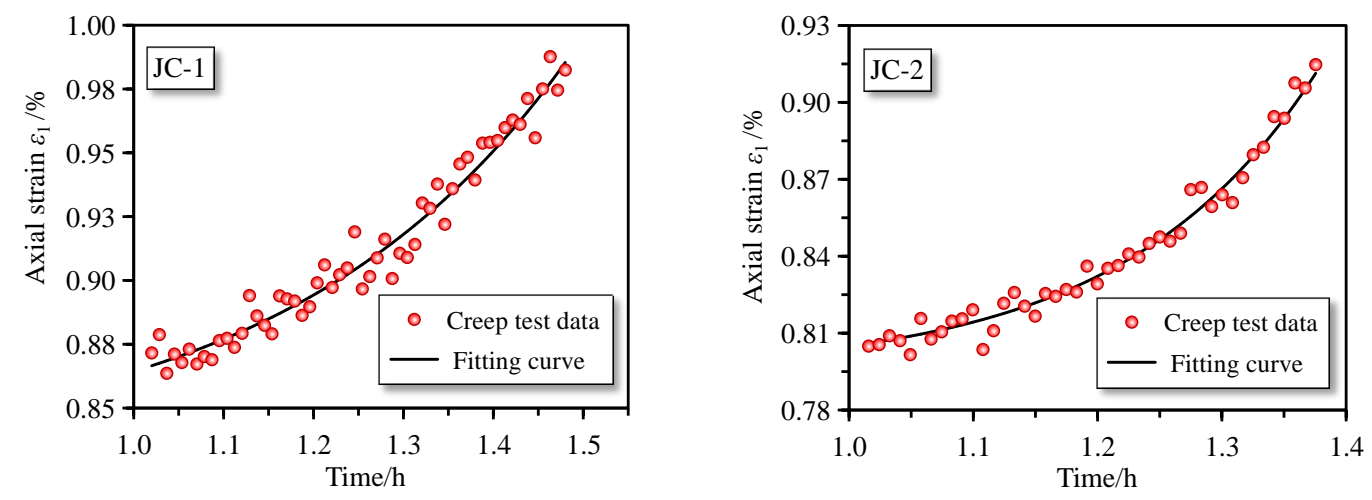

Figure 9. Comparison between creep test data and fitting curves of accelerating creep stage of coal specimens.

Table 3. Parameter identification of accelerating creep stage

\begin{tabular}{ccccc}
\hline Specimen & $\varepsilon_{0}$ & $\boldsymbol{A}$ & $\boldsymbol{n}$ & $\boldsymbol{R}^{\mathbf{2}}$ \\
\hline JC-1 & 0.8306 & $1.658 \times 10^{-3}$ & 3.1724 & 0.9656 \\
JC-2 & 0.7946 & $2.24 \times 10^{-5}$ & 6.4569 & 0.9765 \\
\hline
\end{tabular}

Table 3 shows that the square of correlation coefficient $R^{2}$ of the fitting results reaches up to 0.96, demonstrating that the use of the exponential function is a feasible method for describing the accelerating creep stage curve of coal. Hence, this non-Newtonian component with exponential change law can be used instead of the ideal viscous component of the classical Nishihara model, which has a constitutive equation as follows:

$$
\sigma=\eta(t) \frac{\mathrm{d} \varepsilon}{\mathrm{d} t}=\eta(t) \dot{\varepsilon}
$$

Coal strain can be calculated by integrating Equation (3) as follows:

$$
\varepsilon=\frac{\sigma}{\eta(t)} t+C
$$

The non-Newtonian component expression can be obtained by equating Equation (2) to Equation (3) and taking $A=\sigma / \eta_{0}, C=\varepsilon_{0}$ as follows:

$$
\eta(t)=\frac{\eta_{0} t}{\exp (n t)}
$$

where $\eta(t)$ and $\eta_{0}$ are the nonlinear and initial viscosity coefficients of non-Newtonian unit, respectively. 


\subsubsection{Introduction of Negative Elastic Modulus $E_{3}$ (Shear Modulus $G_{3}$ )}

For the rheological mechanics of rock engineering, when the visco-elastic flow develops to a certain extent, it will transform into a visco-plastic flow state. The correlation research indicates that accelerating creep may occur in coal and other strain-softening materials [25]. Thus, the elastic potential energy will gradually decrease as creep deformation develops. At this time, the flow rule of coal and rock mass should be similar to that of viscoelastic-plastic flow. Thus, a negative elastic modulus, $E_{3}$ (corresponding to the shear modulus $G_{3}$ under 3D stress condition), can be introduced parallel with the viscoplastic component to construct a new nonlinear viscoelastic-plastic body (Figure 10). This viscoelastic-plastic body characterizes the yield that weakens with the increase in coal strain in the accelerating creep stage. It also indicates the process of releasing elastic potential energy.

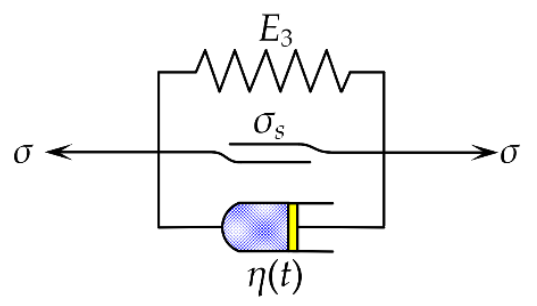

$E_{3}$ is the elastic modulus;

$\sigma_{s}$ is yield stress;

$\eta(t)$ is the nonlinear viscosity coefficient.

Figure 10. Nonlinear viscoelastic-plastic body.

According to a series of rules, the nonlinear viscoelastic-plastic body in the one-dimensional (1D) case obeys the following relationships:

$$
\left\{\begin{array}{l}
\varepsilon=\varepsilon_{1}=\varepsilon_{2}=\varepsilon_{3} \\
\sigma=\sigma_{1}+\sigma_{2}+\sigma_{3}
\end{array}\right.
$$

Consequently, the constitutive relation of this nonlinear viscoelastic-plastic body is described as follows:

$$
\sigma-\sigma_{s}=E_{3} \varepsilon+\eta(t) \dot{\varepsilon} .
$$

On the basis of the initial condition, $t=0, \varepsilon=0$, the creep solution of the nonlinear viscoelastic-plastic body can be obtained by integrating constitutive Equation (7):

$$
\varepsilon=\frac{\sigma-\sigma_{s}}{E_{3}}\left[1-\exp \left(-\frac{E_{3}}{\eta(t)} t\right)\right]=\frac{\sigma-\sigma_{s}}{E_{3}}\left\{1-\exp \left[-\frac{E_{3}}{\eta_{0}} \exp (n t)\right]\right\} .
$$

The creep rate and creep acceleration of the nonlinear viscoelastic-plastic body are solved by taking the first and the second derivatives of Equation (8) as follows:

$$
\left\{\begin{array}{l}
\dot{\varepsilon}=n \frac{\sigma-\sigma_{s}}{\eta_{0}} \exp \left[n t-\frac{E_{3}}{\eta_{0}} \exp (n t)\right] \\
\ddot{\varepsilon}=n^{2} \frac{\sigma-\sigma_{s}}{\eta_{0}} \exp \left[n t+1-2 \frac{E_{3}}{\eta_{0}} \exp (n t)\right]
\end{array},\right.
$$

where $\dot{\varepsilon}$ is the creep rate and $\ddot{\varepsilon}$ is the creep acceleration.

Equations (8) and (9) show that strain $\varepsilon$ is the function of time $t$ during the coal creep process. Only when $E_{3}<0$ can the creep rate and creep acceleration be above zero, because the acceleration index $n$, yield stress $\sigma_{s}$, and nonlinear viscosity coefficient $\eta(t)$ are the constants that are greater than zero, indicating that the strain of the nonlinear viscoelastic-plastic body will increase over time. Therefore, $E_{3}<0$ not only has mathematical significance, but also has definite physical meaning that describes the process of releasing elastic potential energy in the accelerating creep stage. Therefore, this non-linear viscoelastic-plastic unit can be used to capture the changing regularity of accelerating creep. 


\subsection{Nonlinear Viscoelastic-Plastic Creep Model}

The nonlinear viscoelastic-plastic body in Figure 10 can be replaced by the ideal viscoplastic body in the classical Nishihara model to establish a new nonlinear viscoelastic-plastic creep model with six elements, because its accelerating creep characteristics are improved. Figure 11 shows the improved method for describing the non-decay creep characteristic of coal mass.

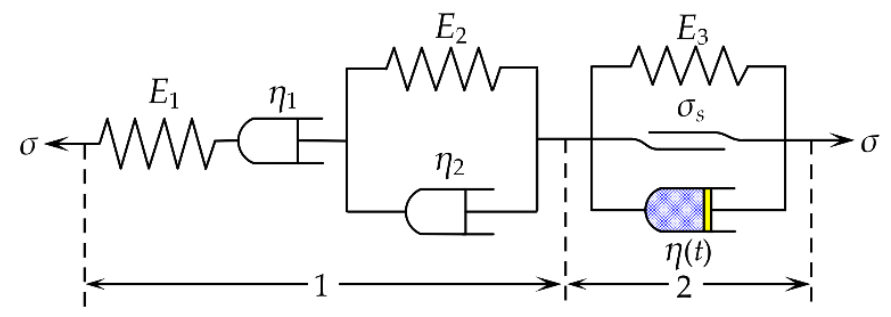

$E_{1}, E_{2}$ and $E_{3}$ are the elastic modulus; $\eta_{1}$ and $\eta_{2}$ are the elastic modulus; $\sigma_{s}$ is yield stress; $\eta(t)$ is the nonlinear viscosity coefficient.

Figure 11. Improved nonlinear viscoelastic-plastic creep model

According to the improved model in Figure 11, if the value of constant stress $\sigma$ is less than $\sigma_{s}$, then the model is in a viscoplastic state, that is, Part 2, is rigid and only Part 1 deforms in the model. Therefore, the improved model degenerates into a generalized Kelvin body. However, if the constant stress $\sigma$ is more than $\sigma_{s}$, Part 1 and Part 2 both deform in the model, and the nonlinear viscoelastic-plastic creep model meets the following formulation:

$$
\left\{\begin{array}{ll}
\varepsilon=\varepsilon_{1}+\varepsilon_{2}+\varepsilon_{3}, & \sigma=\sigma_{1}=\sigma_{2}=\sigma_{3} \\
\sigma_{1}=E_{1} \varepsilon_{1} & \sigma_{2}=E_{2} \varepsilon_{2}+\eta_{2} \dot{\varepsilon}_{2} \\
\sigma_{3}=\sigma_{s}+E_{3} \varepsilon_{3}+\eta(t) \dot{\varepsilon}_{3} &
\end{array} .\right.
$$

The creep equations of the improved model under 1D stress state can be expressed on the basis of Equation (10) as follows:

$$
\left\{\begin{array}{ll}
\varepsilon=\frac{\sigma}{E_{1}}+\frac{\sigma}{E_{2}}\left[1-\exp \left(-\frac{E_{2}}{\eta_{1}} t\right)\right], & \sigma<\sigma_{\mathcal{S}} \\
\varepsilon=\frac{\sigma}{E_{1}}+\frac{\sigma}{E_{2}}\left[1-\exp \left(-\frac{E_{2}}{\eta_{1}} t\right)\right]+\frac{\sigma-\sigma_{s}}{E_{3}}\left\{1-\exp \left[-\frac{E_{3}}{\eta_{0}} \exp (n t)\right]\right\}, & \sigma \geq \sigma_{\mathcal{S}}
\end{array} .\right.
$$

Underground coal and rock masses are usually subject to complex 3D stress states. Therefore, deducing the creep model under 3D conditions is of great engineering significance. In the 3D stress state, the coal stress tensor $\sigma_{i j}$ can be decomposed as a spherical stress tensor $\sigma_{m}$ and deviatoric stress tensor $S_{i j}$, which can be represented as follows [26]:

$$
\sigma_{i j}=S_{i j}+\delta_{i j} \sigma_{m}
$$

where $\delta_{i j}$ is the Kronecker function.

Generally, a spherical stress tensor $\sigma_{m}$ only changes the volume of an object, and deviatoric stress tensor $S_{i j}$ only changes the shape. Similarly, strain tensor $\varepsilon_{i j}$ can also be divided into spherical strain tensor $\varepsilon_{m}$ and partial strain tensor $e_{i j}$. Thus, $\varepsilon_{m}$ and $e_{i j}$ are given by the following:

$$
\varepsilon_{i j}=e_{i j}+\delta_{i j} \varepsilon_{m}
$$

Typically, coal and rock masses can be regarded as isotropic media in the mechanical analysis. On the basis of the broad sense of Hook's law, the constitutive relationship under the 3D stress state can be defined as follows:

$$
\sigma_{m}=3 K \varepsilon_{m}, \quad S_{i j}=2 G e_{i j},
$$

where $K$ and $G$ are the bulk and shear moduli, respectively. 
For simplicity, we assumed that the creep deformation is only caused by deviatoric stress tensor $S_{i j}$. Thus, the 3D creep equation can be described as follows:

$$
\left\{\begin{array}{ll}
\varepsilon=\frac{S_{i j}}{2 G_{1}}+\frac{S_{i j}}{2 G_{2}}\left[1-\exp \left(-\frac{G_{2}}{\eta_{1}} t\right)\right], & \sigma<\sigma_{s} \\
\varepsilon=\frac{S_{i j}}{2 G_{1}}+\frac{S_{i j}}{2 G_{2}}\left[1-\exp \left(-\frac{G_{2}}{\eta_{1}} t\right)\right]+\frac{\sigma-\sigma_{s}}{2 G_{3}}\left\{1-\exp \left[-\frac{G_{3}}{\eta_{0}} \exp (n t)\right]\right\}, & \sigma \geq \sigma_{s}
\end{array} .\right.
$$

However, most conventional triaxial compression tests are conducted under constant confining pressure conditions, in which case $\sigma_{2}=\sigma_{3}$. By substituting $S_{i j}=\left(\sigma_{2}-\sigma_{3}\right)$ into Equation (14), the axial strain in nonlinear viscoelastic-plastic creep model can be deduced as follows:

$$
\left\{\begin{array}{ll}
\varepsilon=\frac{\sigma_{1}-\sigma_{3}}{3 G_{1}}+\frac{\sigma_{1}-\sigma_{3}}{3 G_{2}}\left[1-\exp \left(-\frac{G_{2}}{\eta_{1}} t\right)\right], & \sigma<\sigma_{\mathcal{S}} \\
\varepsilon=\frac{\sigma_{1}-\sigma_{3}}{3 G_{1}}+\frac{\sigma_{1}-\sigma_{3}}{3 G_{2}}\left[1-\exp \left(-\frac{G_{2}}{\eta_{1}} t\right)\right]+\frac{\left(\sigma_{1}-\sigma_{3}\right)-\sigma_{s}}{3 G_{3}}\left\{1-\exp \left[-\frac{G_{3}}{\eta_{0}} \exp (n t)\right]\right\}, & \sigma \geq \sigma_{\mathcal{S}}
\end{array} .\right.
$$

\section{Model Validation and Parametric Analysis}

\subsection{Model Validation}

At present, the main methods used to determine model parameters include regression analysis, least squares, rheological curve decomposition, and the optimization separation method [27]. Among these methods, the least squares method is the most widely used in identifying creep parameters. However, it cannot perfectly address nonlinear problems. Thus, the final result will not converge or may converge to the local minimum if the selection of the iterative initial value is unreasonable. In addition, this method has a slow convergence rate. For this reason, the universal global optimization algorithm (UGO) in 1stop analysis software was used in this study to fit the non-decay creep curves in coal creep tests. The greatest advantage of this method is that the initial value of parameters is randomly given by the program, and the optimal solution can be found through its UGO algorithm, which can avoid the selection of initial parameters, thus enabling the rapid and precise identification of the model parameters [28].

Because the non-linear viscoelastic-plastic creep model proposed in this work was improved for the accelerating creep characteristics, the experimental data under $45 \mathrm{MPa}$ with non-decay creep characteristics were used to validate the improved model using 1stop in accordance with the second item $\left(\sigma \geq \sigma_{s}\right)$ in Equation (15). Meanwhile, the fitting results were also compared with the classical Nishihara model. The parameter fitting results of different creep models are listed in Table 4, and the comparison results of the fitting curves and test data are shown in Figure 12.
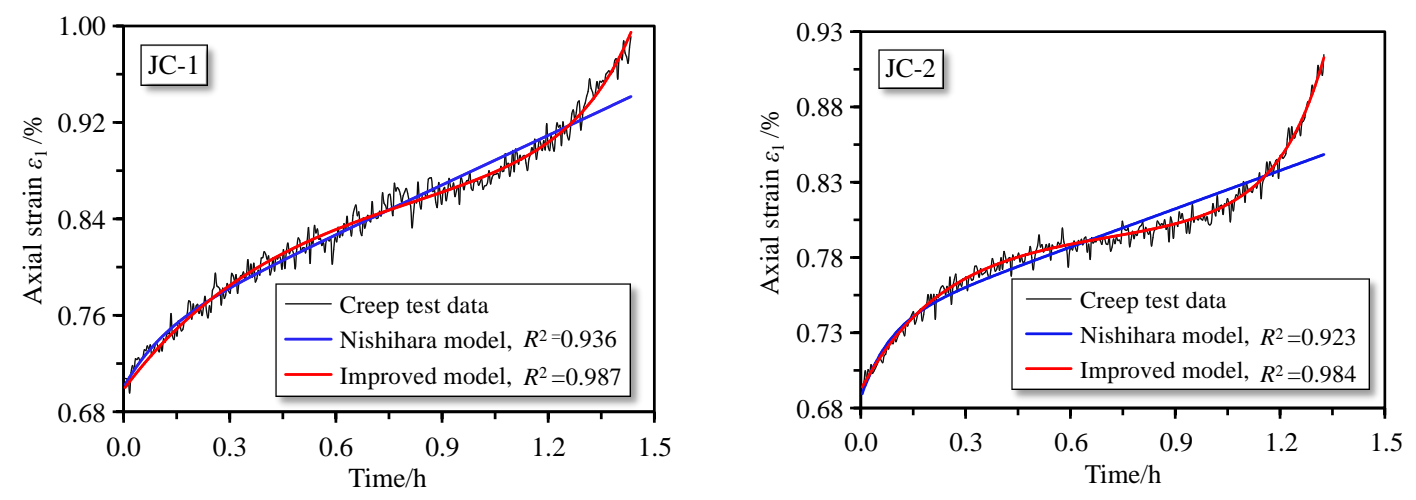

Figure 12. Comparisons between fitting curves of different creep models and experimental data. 
Table 4. Parameter fitting results of different creep models.

\begin{tabular}{ccccccccccc}
\hline Model & Specimen & $G_{1} / \mathrm{GPa}$ & $G_{2} / \mathrm{GPa}$ & $G_{3} / \mathrm{GPa}$ & $\eta_{1} /(\mathrm{GPa} \cdot \mathbf{h})$ & $\eta_{0} /(\mathrm{GPa} \cdot \mathbf{h})$ & $\eta_{2} /(\mathrm{GPa} \cdot \mathbf{h})$ & $n$ & $S_{h} / \mathbf{M P a}$ & $\boldsymbol{R}^{2}$ \\
\hline Improved & $\mathrm{JC}-1$ & 20.080 & 86.918 & -164.902 & 37.604 & 451.877 & - & 1.507 & 41.027 & 0.987 \\
model & $\mathrm{JC}-2$ & 21.213 & 144.289 & -11.506 & 34.096 & 8.211 & - & 1.114 & 43.991 & 0.984 \\
\hline Nishihara & $\mathrm{JC}-1$ & 20.815 & 455.497 & - & 47.673 & - & 0.010 & - & 43.999 & 0.936 \\
model & $\mathrm{JC}-2$ & 21.326 & 305.679 & - & 24.715 & - & 0.361 & - & 43.969 & 0.923 \\
\hline
\end{tabular}

Notes: $G_{1}, G_{2}$, and $G_{3}$ are the shear modulus; $\eta_{1}$ and $\eta_{2}$ are the viscosity coefficient, respectively; $\eta_{0}$ and $\eta(t)$ are the initial and nonlinear viscosity coefficients, respectively; $n$ is the acceleration index; $S_{h}$ is the deviatoric yield stress of the model.

Table 4 shows that all the squares of correlation coefficient $R^{2}$ of the nonlinear viscoelastic-plastic creep model are above 0.98 , which is higher than those of the classical Nishihara model, thereby showing good agreement with the experimental data. Combining the comparison results in Figure 12, the classical Nishihara model can reflect the decelerating creep and steady state creep stages, but the fitting curves still maintain a linear tendency during the accelerating creep, thus deviating from the experimental data and resulting in a large discrepancy. By contrast, the fitting curves obtained by the improved model basically coincide with the experimental data and exhibit an exponential increasing law that can describe the full creep stage of coal, especially the accelerating creep stage, in which the coal strain gradually increased with time. Furthermore, the fitting results demonstrate the high applicability and superiority of the proposed nonlinear viscoelastic-plastic creep model.

\subsection{Parametric Sensitivity Analysis}

A negative elastic modulus $E_{3}$ (corresponding to the shear modulus $G_{3}$ ) and a non-Newtonian component with exponential change laws are introduced in the improved model. The introduction is necessary to describe the non-decay creep characteristics of coal, among which the negative elastic modulus $E_{3}$ represents the release of elastic potential energy during the accelerating creep stage. In addition, the acceleration creep index $n$ of the non-Newtonian component can embody the accelerated creep rate. To further analyze the sensitive effect of $n$ value on coal creep deformation, the creep acceleration equation can be deduced from Equation (15), as shown in Equation (16). With specimen JC-2 as a sample, the creep curves and creep acceleration curves with different acceleration creep indexes $n\left(n=n_{1}, n_{2}, n_{3}, n_{4}, n_{5}\right)$ are shown in Figure 13.

$$
\ddot{\varepsilon}=-\frac{\left(\sigma_{1}-\sigma_{3}\right) G_{2}}{3 \eta_{1}^{2}} \exp \left(-\frac{G_{2}}{\eta_{1}} t\right)+n^{2} \frac{\left[\left(\sigma_{1}-\sigma_{3}\right)-\sigma_{s}\right]}{3 \eta_{0}} \exp \left[n t+1-\frac{2 G_{3}}{\eta_{0}} \exp (n t)\right], \quad \sigma \geq \sigma_{s}
$$

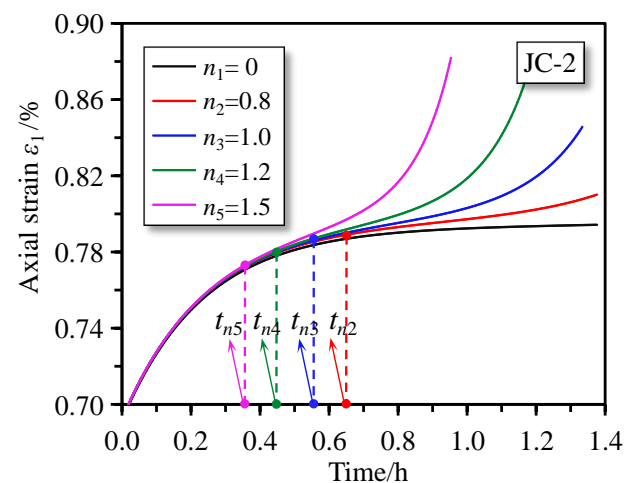

(a) Creep curves

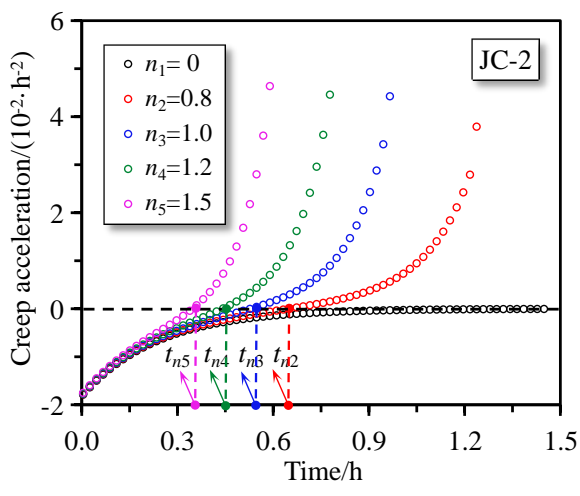

(b) Creep acceleration curves

Figure 13. Creep and creep acceleration curves with different accelerating creep exponents $n$. 
Figure 13a shows that when $n=0$, the relationship between axial strain and creep time is linear. Thus, the non-Newtonian component degenerates into an ideal Newtonian unit. While $n>0$, the concavity-convexity of the coal creep curve will be changed, and the axial strain increases nonlinearly and presents non-decay creep characteristic. When the value of acceleration creep index $n$ is large, the increasing rate of coal strain is fast, and the corresponding creep inflection point also appears early.

Subsequently, further analysis on creep acceleration in accordance with Figure 13b shows that if $n=0$, then creep acceleration gradually increases to the zone, that is, $\lim _{t \rightarrow \infty} \ddot{\varepsilon}=0$. However, if $n>0$, then the creep acceleration of coal tends to be infinite along with time in the non-decay creep process, that is, $\lim _{t \rightarrow \infty} \ddot{\varepsilon}=+\infty$. The corresponding creep inflection point only appears when $\ddot{\varepsilon}=0$. The creep instability condition, that is, $\ddot{\varepsilon}>0$, suggests that coal will fail once it enters into the accelerating creep stage. In other words, the value of accelerating creep index $n$ is directly proportional to the instability level, that is, a large $n$ value results in the early occurrence of coal failure.

If we can accurately predict the occurrence time of the instability failure of coal and take effective protective measures in time during the actual underground production process, then various geologic hazards and potentially fatal accidents can be reduced to a large extent. The aforementioned analysis suggests that the linear viscoelastic-plastic creep model proposed in this study can accurately reflect the non-decay creep characteristics of coal. Thus, the occurrence time of the creep inflection point can be predicted via model calculation. If the creep inflection point does not appear, then coal instability will not occur. Conversely, once the creep inflection point emerges, the accelerating creep of coal will happen. Then, the occurrence time can be deduced to judge whether or not coal failure, which is crucial for the prevention and control of coal and rock dynamic disasters, will happen.

\section{Conclusions}

This study aims at developing a nonlinear model to describe the viscoelastic-plastic creep behavior of coal. The proposed model consisted of a generalized Kelvin and improved viscoelastic-plastic body using the method of empirical equation and component combination. The validity of the proposed model and the sensitivity of the model parameters were verified and analyzed on the basis of the experimental data. The main conclusions of this study are presented as follows:

(1) A series of multistage creep tests was performed on coal specimens with different stress levels and found that the concavity-convexity in creep curves will be changed when coal enters accelerating creep stage. That is, a creep inflection point is found on the creep curve. If the occurrence time of the creep inflection point can be accurately predicted, then some effective protective measures can be taken in time to avoid the potential safety hazards caused by coal instability and failure.

(2) The exponential function was used to fit the accelerating creep data of coal specimens and achieved the desired results. Thus, a non-Newtonian component was proposed to replace the ideal Newtonian component. Furthermore, a negative elastic modulus $E_{3}$ (shear modulus $G_{3}$ ) was introduced to establish a nonlinear viscoelastic-plastic body to describe the accelerating creep characteristics of coal. Therefore, a new nonlinear viscoelastic-plastic creep model that consists of a generalized Kelvin and improved viscoelastic-plastic body was proposed, and the creep equation of the improved model under constant confining pressure was also derived.

(3) 1 stop analysis software was used to fit the experimental data of the non-decay creep characteristics in the coal multistage creep tests based on the proposed nonlinear viscoelastic-plastic creep model. The results show that the fitting correlation coefficients have $R^{2}$ values above 0.98 , and the fitting curves are highly consistent with the experimental data, which can accurately describe the non-decay creep characteristic of coal and verify the correctness and applicability of the model.

(4) The analyses of creep rate and creep acceleration curves using different acceleration creep indexes $n$ show that if $n=0$, then the non-Newtonian component will degenerate into the ideal Newtonian body, and only the decelerating and steady-state creeps of coal will occur. If $n>0$, then coal 
specimens will present non-decay creep characteristic. When the acceleration creep index $n$ is large, the level of creep acceleration increases, and the occurrence of coal instability appears earlier.

Author Contributions: J.Z. contributed to the conception of the study and designed the experiments; B.L. performed the data analyses; C.Z. wrote the manuscript and P.L. provided fund support.

Funding: The authors gratefully acknowledge the financial support of the National Science Foundation of China under Grant No. 51604096, 51804355, and 51804356. The work in this paper is also supported by the Hebei State Key Laboratory of Mine Disaster Prevention (KJZH2017K08) and Open Fund of Energy Platform Laboratory Project (G201609).

Conflicts of Interest: The authors declare no conflict of interest.

\section{References}

1. Xie, H.P.; Gao, F.; Ju, Y. Research and development of rock mechanics in deep ground engineering. Chin. J. Rock Mech. Eng. 2015, 34, 2161-2178.

2. Yang, S.Q.; Chen, M.; Jing, H.W.; Chen, K.F.; Meng, B. A case study on large deformation failure mechanism of deep soft rock roadway in Xin'An coal mine, China. Eng. Geol. 2017, 217, 89-101. [CrossRef]

3. Wei, S.; Qi, Z.; Luan, Y.L.; Zhang, X.P. A study of surface subsidence and coal pillar safety for strip mining in a deep mine. Environ. Earth Sci. 2018, 77, 627.

4. Xu, J.H.; Miao, X.X.; Zhang, X.C. Analysis of the time-dependence of the coal pillar stability. J. Chin. Coal Soc. 2005, 30, 433-437.

5. Li, Q.; Shi, W.; Yang, R. Deformation mechanisms in a coal mine roadway in extremely swelling soft rock. SpringerPlus 2016, 5, 1310. [CrossRef]

6. Liu, C.; Zhou, F.B.; Yang, K.K.; Xiang, X.; Liu, Y.K. Failure analysis of borehole liners in soft coal seam for gas drainage. Eng. Fail. Anal. 2014, 42, 274-283. [CrossRef]

7. Chen, L.; Wang, E.Y.; Ou, J.C.; Fu, J.W. Coal and gas outburst hazards and factors of the No. B-1 Coalbed, Henan, China. Geosci. J. 2017, 22, 171-182. [CrossRef]

8. Kim, D.K. Comparisons of overstress theory with an empirical model in creep prediction for cohesive soils. KSCE J. Civ. Eng. 2005, 9, 489-494. [CrossRef]

9. Xia, C.C.; Jin, L.; Guo, R. Nonlinear theoretical rheological model for rock: a review and some problems. Chin. J. Rock Mech. Eng. 2011, 30, 454-463.

10. Zhang, X.D.; Li, Y.J.; Zhang, S.G.; Huo, B.R. Creep study of soft rock and its engineering application. Chin. J. Rock Mech. Eng. 2004, 23, 1635-1639.

11. Ma, K.; Wan, X.L.; Jia, W.F.; Wan, C.H. Advances in rock creep model research and discussion on some issues. Coal Geol. Chin. 2011, 23, 43-47. [CrossRef]

12. Yang, Z.W.; Jin, A.B.; Zhou, Y.; Yan, Q.; Wang, K.; Gao, Y.J. Parametric analysis of Burgers model and creep properties of rock with particle flow code. Rock Soil Mech. 2015, 36, 240-248.

13. Shao, J.F.; Zhu, Q.Z.; Su, K. Modeling of creep in rock materials in terms of material degradation. Comput. Geotech. 2003, 30, 549-555. [CrossRef]

14. Shao, J.F.; Chau, K.T.; Feng, X.T. Modeling of anisotropic damage and creep deformation in brittle rocks. Int. J. Rock Mech. Min. Sci. 2006, 43, 582-592. [CrossRef]

15. Ping, C.; Wen, Y.D.; Wang, Y.X.; Yuan, H.P.; Yuan, B.X. Study on nonlinear damage creep constitutive model for high-stress soft rock. Environ. Earth Sci. 2016, 75, 900.

16. Kang, J.H.; Zhou, F.B.; Liu, C.; Liu, Y.K. A fractional nonlinear creep model for coal considering damage effect and experimental validation. Int. J. Nonlinear Mech. 2015, 76, 20-28. [CrossRef]

17. Cai, T.; Feng, Z.; Jiang, Y. An improved hardening-damage creep model of lean coal: A theoretical and experimental study. Arab. J. Geosci. 2018, 11, 645. [CrossRef]

18. Cai, T.T.; Feng, Z.C.; Zhao, D.; Jiang, Y.L. A creep model for lean coal based on hardening-damage mechanism. Rock Soil Mech. 2018, 39, 70-77.

19. Wang, L.J.; Zhou, H.W.; Rong, T.L.; Ren, W.G. Research on experimental and nonlinear creep constitutive model of coal at depth. J. Chin. Coal Soc. 2018, 43, 110-116. 
20. Ömer, A.; Ito, T.; Özbay, U.; Kwasniewski, M.; Shariar, K.; Okuno, T.; Özgenoğlu, A.; Malan, D.F.; Okada, T. ISRM suggested methods for determining the creep characteristics of rock. Rock Mech. Rock Eng. 2014, 47, 275-290.

21. The Professional Standards Compilation Group of Peoples Republic of China. DL/T5368-2007 Code for Rock Tests of Hydroelectric and Water Conservancy Engineering[S]; Water and Power Press: Beijing, China, 2007.

22. Yang, Y.; Li, Y.; Zhou, X.K. Study on the coal creep test based on the improved Nishihara model. J. Chin. Coal Soc. 2014, 39, 2190-2194.

23. Liu, H.Z.; Xie, H.Q.; He, J.D.; Xiao, M.L.; Zhuo, L. Nonlinear creep damage constitutive model for soft rocks. Mech. Time Depend. Mater. 2016, 21,1-24. [CrossRef]

24. Tao, B.; Wu, F.Q.; Guo, G.M.; Zhou, R.G. Flexibility of visco-elastoplastic model to rheological characteristics of rock and solution of rheological parameter. Chin. J. Rock Mech. Eng. 2005, 24, 3165-3171.

25. Cao, S.G.; Bian, J.; Li, P. Rheologic constitutive relationship of rocks and a modifical model. Chin. J. Rock Mech. Eng. 2002, 21, 632-634.

26. Jiang, Q.; Qi, Y.; Wang, Z.; Zhou, C. An extended Nishihara model for the description of three stages of sandstone creep. Geophys. J. Int. 2013, 193, 841-854. [CrossRef]

27. Cao, L.; Lu, H.F.; Yao, D.X.; Zhou, L.; Xu, Z.D. Research summary of rock creep model and creep parameter identification. Open J. Nat. Sci. 2018, 6, 395-400. [CrossRef]

28. Cheng, X. Optimization·Fitting.Modeling: Application Detailed; China Building Materials Press: Beijing, China, 2012; pp. 1-4.

(C) 2019 by the authors. Licensee MDPI, Basel, Switzerland. This article is an open access article distributed under the terms and conditions of the Creative Commons Attribution (CC BY) license (http://creativecommons.org/licenses/by/4.0/). 